\title{
Channel Assignment for Infrastructure-Based 802.11 WLANs
}

\author{
Ya-Yin Yang, I-Yung Chen, and Chu-Sing Yang
}

\begin{abstract}
The scarcity of non-overlapping channels is always an important issue especially in case where access points (APs) are very dense. This paper presents a distributed dynamic channel assignment (DCA) algorithm to improve network throughput and decrease the computation complexity by maximizing the sum of weighted signal to interference plus noise ratio (SINR). The proposed scheme allows using partially overlapping channels instead of non-overlapping channels. Simulation results show the effectiveness of the proposed algorithms and compare the performance with the state-of-the-art DCA.
\end{abstract}

Index Terms-Channel assignment, partially overlapped channels, signal-interference-noise-ratio, WLANs.

\section{INTRODUCTION}

Wireless local area networks (WLANs) have spread so rapidly and developed dramatically in areas such as campuses, homes, public hotspots, airports, and offices. With the dense deployment of access points (APs), the signal interference problem has become more serious. In order to use available resources as efficiently as possible and improve the network throughput, most dynamic channel assignment (DCA) algorithms focus on minimizing the interference to improve the network throughput [1]-[3]. In [1], the authors aim to minimize the maximum effect of interference on clients in all overlap regions between APs. This interference model considers both channel separation and the number of clients affected in an overlapping region. In least congested channel search (LCCS) [2], each AP selects the channel with the least amount of traffic in it. In [3], the authors develop an interference model between overlapping channels by using an overlapping channel interference factor. Such interference is captured through interfering AP transmit power, path loss, and overlapping channel interference factor. The objective is to minimize the total interference at each AP.

Recently, some studies verified the effectiveness of using partially overlapping channels in place of non-overlapping ones in both theoretical analysis and experimentation [1], [3], [4]-[9]. Unlike the previous literature which takes into consideration interference between APs, a channel assignment algorithm based on maximizing total signal to interference plus noise ratio (SINR) at the user level is first proposed in [4]. Cui et al. [5] establish a novel interference model that considers both the channel separation and the

Manuscript received December 20, 2012; revised March 14, 2013. This work was supported in part by the NSC under Grant No. 101-2218-E-006-012.

The authors are with Institute of Computer and Communication Engineering, National Cheng Kung University, Tainan City, Taiwan (R.O.C.) (e-mail: ivyyang64@ gmail.com ben2103gto@ gmail.com, csyang@mail.ee.ncku.edu.tw). physical distance separation of two nodes. The authors prove that maximizing aggregated throughput in the network and minimizing the total weighted interference are equivalent. They propose an approximate algorithm to minimize the total weighted interference. In [6], the authors conclude that minimizing total interference is equal to maximizing system throughput. This minimizing interference problem is formulated as a directed weighted interference graph. Then a greedy algorithm is proposed. In [7], Mishra et al. measure the receiving power among partially overlapping channels and evaluate how partially overlapping channels can be exploited. In [8], the authors have quantified the adjacent channel interference caused by utilization of partially overlapping channels in $802.11 \mathrm{~b} / \mathrm{g}$ networks. In [9], the authors propose a weighted conflict graph to model interference. Based on this model, two algorithms, the greedy algorithm and the genetic algorithm, for partially overlapping channel assignment are proposed

Most of the previous literature on interference models consider interference between two interfering APs, and then minimize interference among APs. In reality, looking at interference from the users' perspective is more efficient for throughput improvement. The data rate is directly proportional to received SINR. In this paper, we propose a distributed DCA algorithm for maximizing the total weighted SINR by using partially overlapping channels in infrastructure-based 802.11 WLANs. Each AP in an area of interest can perform channel assignment alone.

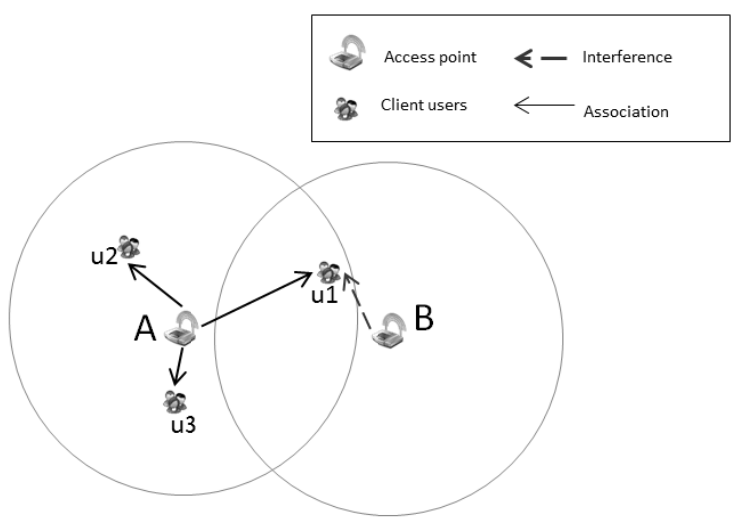

Fig. 1. The user located farthest from the AP

We think that the user who is more vulnerable to interference should have a higher the weight. The users, ul and $\mathrm{u} 4$ would serve the strongest signal interference as Fig. 1 and Fig. 2 shows. The SINR of these users can be very low because of co-channel interference. Conversely, the impact of the co-channel interference on SINR to other users is not obvious. The SINR of these users can be increased if APs choose the suitable channels. We consider two special cases: one is that the user located farthest from the AP has weight 1; 
the other that is the user with the lowest SINR has weight

The user $\mathrm{ul}$ is the farthest user from AP A and close to the interference AP B as Fig. 1 shows. We will give this kind of user highest weight. But the farthest user is not always serving the strongest signal interference. Looking at the Fig. although the user $\mathrm{u} 5$ is the farthest user from the AP A, but there are no inferences close to it. In contrast with user u5, user $\mathrm{u} 4$ may be the user who is serving the strongest signal interference

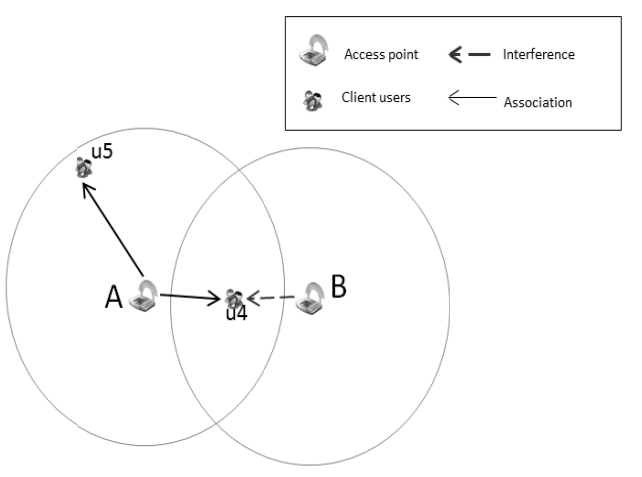

Fig. 2. The user with the lowest SINR

The rest of this paper is formed as follows: The channel assignment model is described in Section II. The performance evaluation and conclusions are presented in Section III and IV, respectively.

\section{PROBLEM Formulation}

In this section, we formulate the DCA problem for IEEE 802.11-based infrastructure networks. Assume there are $K$ channels available in the underlying wireless physical layer. For instance, $K$ is 11 for the IEEE $802.11 \mathrm{~b} / \mathrm{g}$ standard in Taiwan. In IEEE $802.11 \mathrm{~b} / \mathrm{g}$ WLAN, channels 1,6 and 11 are non-overlapping channels, as shown in Fig. 2. The system model considered in this paper consists of $M$ access points (APs) and $N$ client users. Each AP may serve different numbers of users and each user connects to only one AP. The association assignment between users and APs is assumed fixed.

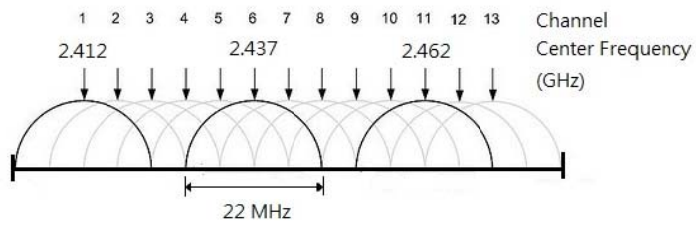

Fig. 3. Wi-Fi channels $(802.11 \mathrm{~b} / \mathrm{g})$

For a given AP $j$, the received SINR at its associated client user $i$ is

$$
\operatorname{SINR}_{i}=\frac{P_{j} g_{i j}}{W+\sum_{m: m \neq j} \gamma(m, j) P_{m} g_{i m}}
$$

where $P_{j}$ is the transmit power of AP $j, W$ is background noise. $g_{i j}$ denotes link gain between user $i$ and AP $j$, including the distance-dependent path loss, shadowing and multipath fading. $\gamma(m, j)$ denotes channel overlapping degree between AP $m$ and AP $j$. The channel overlapping degree is defined as follows [3], [4]:

$$
\gamma(m, j)=\max \left(0,1-\left|C_{m}-C_{j}\right| \times \frac{1}{F}\right)
$$

where $C_{j} \in\{1, \cdots, K\}$ is the channel assigned to $\mathrm{AP} j$ and $F$ is the maximum number of overlapping channels. In IEEE $802.11 \mathrm{~b} / \mathrm{g}$ WLANs, $F$ is equal to 5 . The result of this channel overlapping degree is shown in Table 1. In fact, the channel overlapping degree is not decreasing linearly.

The calculations of the channel overlapping degree for 802.11b WLANs has been shown in [10], and we show it in Table 2. The channel model considered in this paper is only the distance-dependent path loss. Therefore the received SINR at client user $i$ has now become:

$$
\operatorname{SINR}_{i}=\frac{P_{j} d(i, j)^{-n}}{W+\sum_{m: m \neq j} \gamma(m, j) P_{m} d(i, m)^{-n}}
$$

where $d(i, j)$ denotes the distance between user $i$ and AP $j$, and $n$ is the path loss exponent. In general, the path loss exponent can reach values in the range of 2 to 6 [11].

We consider a distributed DCA scheme. It means each AP adjusts channel assignment alone. Each AP chooses a channel to maximize total weighted SINR at the user level. For a given AP $j$, we use $A_{j}$ to denote the set of users that communicate to the AP $j$. Our goal in channel assignment is to maximize the following objective function:

$$
\sum_{i \in A_{j}} w_{i} \operatorname{SINR}_{i}
$$

Therefore, the optimization problem stated in (4) is given as:

$$
\underset{C_{j}}{\operatorname{Max}} \sum_{i \in A_{j}} w_{i} \operatorname{SINR}_{i}
$$

where $w_{i}$ is the weight factor with $0 \leq w_{i} \leq 1$. The user who is more vulnerable to interference should have a higher the weight. Note that (5) is more general than [4], which sets all weight equal to 1 . Intuitively, the farther the distance from AP $j$ for a user $i$ is, the higher the weight will be. We consider the first special case, where we set a weight factor equal to 1 for the farthest users away from $\operatorname{AP} j$, and the others equal to 0 . The farthest users associated with AP $j$ may be more than one. From this point on, we call this special case as Case I.

TABLE I: CHANNEL OVERLAPPING DEGREE

\begin{tabular}{|c|c|c|c|c|c|c|}
\hline $\begin{array}{c}\text { Channel } \\
\text { Separation }\end{array}$ & 0 & 1 & 2 & 3 & 4 & $5-10$ \\
\hline $\begin{array}{c}\text { Overlappi } \\
\text { ng Degree }\end{array}$ & 1 & 0.8 & 0.6 & 0.4 & 0.2 & 0 \\
\hline
\end{tabular}

TABLE II: CHANNEL OVERLAPPING DEGREE

\begin{tabular}{|c|c|c|c|c|}
\hline $\begin{array}{c}\text { Channel } \\
\text { Separation }\end{array}$ & 0 & 1 & 2 & 3 \\
\hline $\begin{array}{c}\text { Overlapping } \\
\text { Degree }\end{array}$ & 1 & 0.7272 & 0.2714 & 0.0375 \\
\hline $\begin{array}{c}\text { Channel } \\
\text { Separation }\end{array}$ & 4 & 5 & 6 & $7-10$ \\
\hline $\begin{array}{c}\text { Overlapping } \\
\text { Degree }\end{array}$ & 0.0054 & 0.0008 & 0.0002 & 0 \\
\hline
\end{tabular}


Actually, the user farthest away AP $j$ is not necessarily subject to the strong interference because it may be far from interference source. Hence, we consider another special case, where we set weight factor equal to 1 for the users with lowest SINR, and the others equal to 0 . We call this case as case II.

We propose the algorithm for Case I that each AP can execute it in a distributed way as shown below in Algorithm 1.
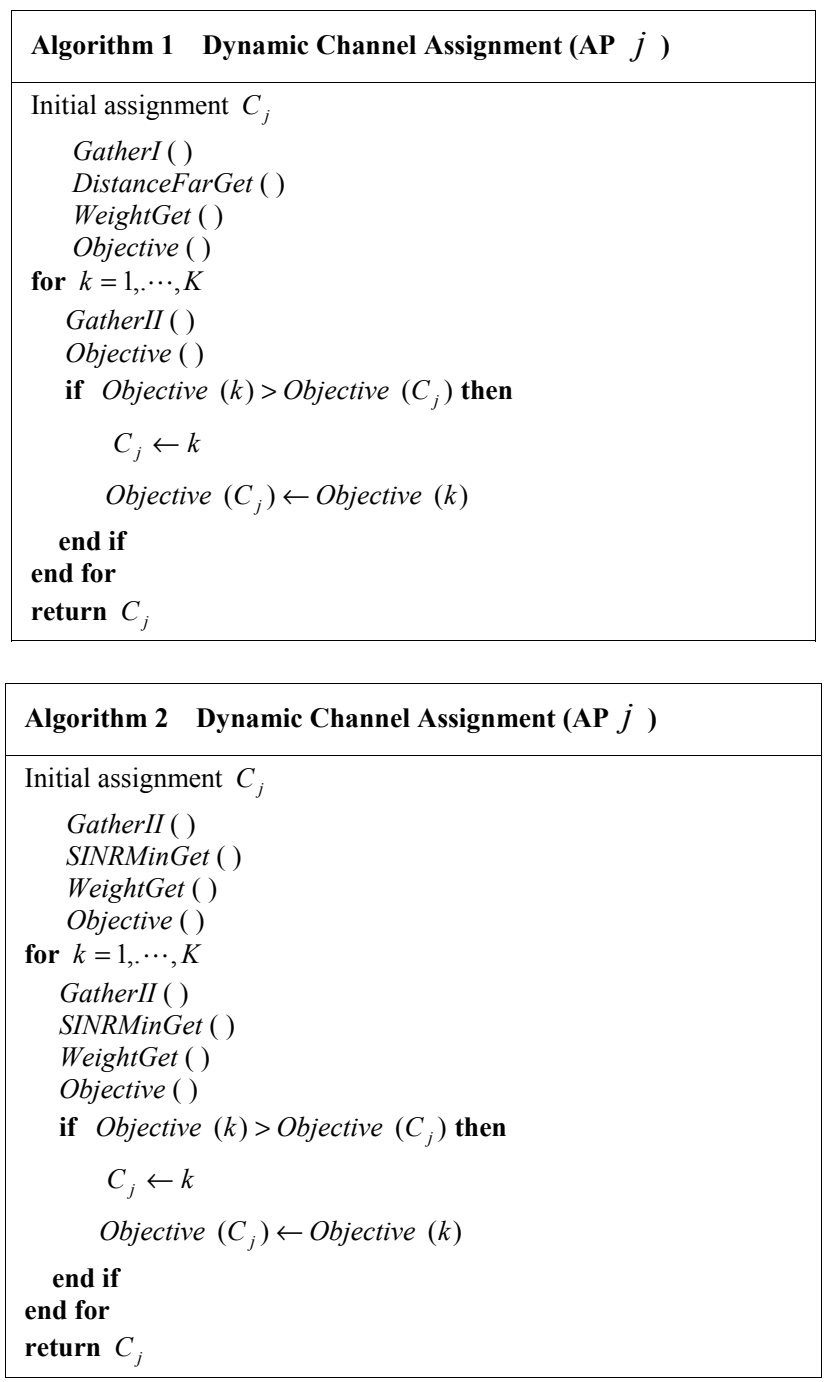

GatherI ( ) is a procedure that is used to gather SINR statistic and location information from each associated user. DistanceFarGet ( ) is a procedure that the AP $j$ computes distance between itself and its users to decide which user is farthest away $\operatorname{AP} j$. WeightGet ( ) is a weight assignment procedure. Objective ( ) computes objective function (4). GatherII ( ) needs to gather SINR statistic which is reported by each associated user.

Next, we propose the algorithm for Case II that each AP can execute it in a distributed way as depicted in Algorithm 2. SINRMinGet ( ) is a procedure to decide which user has lowest SINR. Because different channel assignment will produce different SINR value, the user with lowest SINR may be different. Hence, SINRMinGet ( ) and WeightGet ( ) are included in loop.

After assigning the channel, we calculate the data rate $R$ of user $i$ by Shannon's capacity theorem [11].

$$
R_{i}=B \times \log _{2}\left(1+\operatorname{SINR}_{i}\right)
$$

where $B$ is the physical channel bandwidth in Hertz. The total throughput of AP $j$ is the sum of data rates of all users associated with AP $j$. In the next section, we will compare the total throughput and the minimum user SINR of each AP by using our DCA and the other three channel assignment, namely the AklMaxSINR [4], Hminmax [1] and all APs assigned in the same channel (AASC).

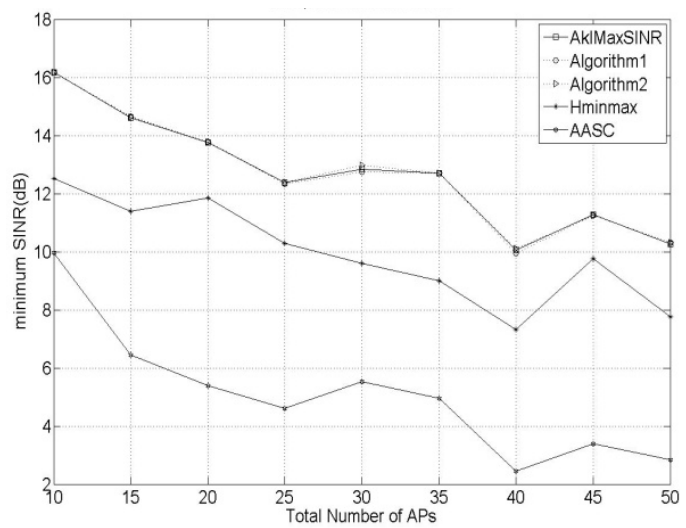

Fig. 3. Comparison of minimum SINR

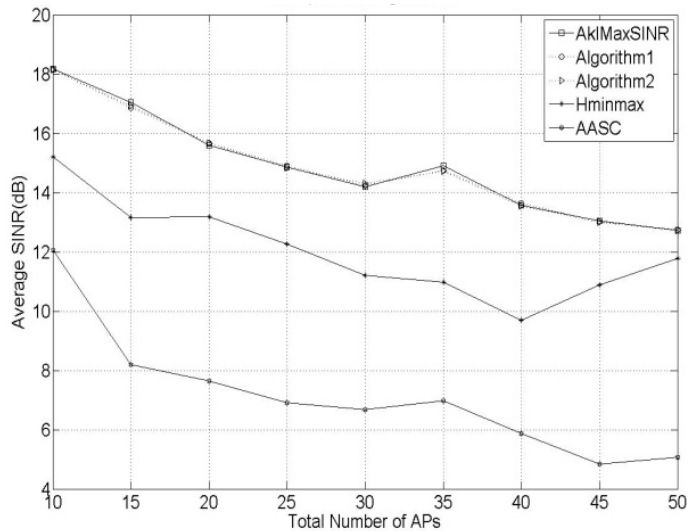

Fig. 4. Comparison of average SINR

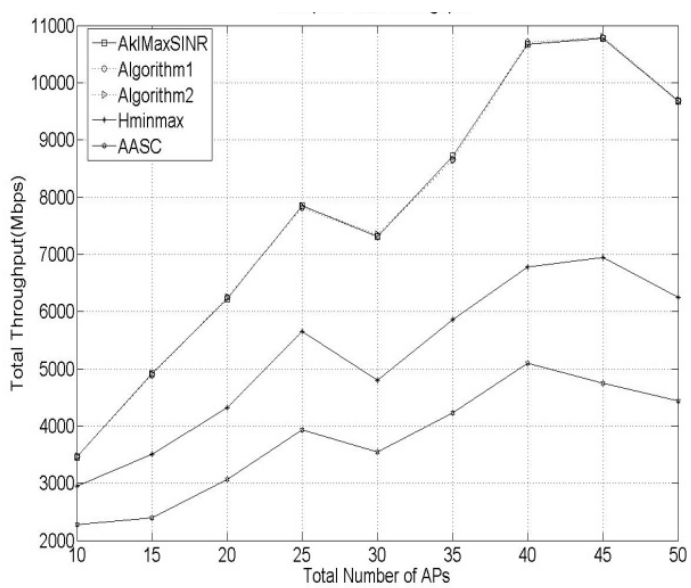

Fig. 5. Comparison of total throughput

\section{Performance Evaluation}

In this section, we verify our DCA algorithms effectiveness by great quantity simulations. We will compare 
performance with the other algorithms which are related to the DCA that considers partially overlapping channel assignment.

\section{A. Simulation Configurations}

The APs and users are distributed over a three dimensional $100 m \times 100 m \times 100 m$ region and are chosen uniformly in a random manner for the experiment. The network topologies contain 10-50 APs. The associated users are randomly generated within $10 m \times 10 m \times 10 m$ range around an AP. The number of users associated with an AP is from 1 to 6 and is equally likely. Initially, all APs are in the same channel, and transmit power is set to $100 \mathrm{~mW}$. The receiver sensitivity threshold is $-89 \mathrm{dBm}$. The available channels are 11 . The pathloss exponent is 2 . The channel bandwidth is set to 20 $\mathrm{MHz}$. We present numerical simulation results, which are averaged over 100 runs.

\section{B. Comparison of Minimum SINR}

Fig. 3 is the minimum user SINR comparison with different numbers of APs by using channel overlapping degrees in Table 2. From the simulation result shows in the Fig. 3, we can clearly see the minimum user SINR by using our algorithms are anticipated to perform well than the Hminmax and the AASC. It was found that our algorithms achieve SINR improvement by at least $1.8 \mathrm{~dB}$ and $6 \mathrm{~dB}$ compared to the Hminmax and the AASC algorithms, respectively. Another observation from Fig. 3 is that our proposals are perform as well as to the AklMaxSINR, but computation complexity of our algorithms is $O(K)$. However, the AklMaxSINR computation complexity is up to $O\left(K^{M}\right)$.

\section{Comparison of Average SINR}

Fig. 4 shows average SINR of all users plotted against the number of APs by using channel overlapping degrees in Table 2. The average SINR was improved by at least $1 \mathrm{~dB}$ and $6 \mathrm{~dB}$ compared to the Hminmax and the AASC algorithms, respectively. The average SINR performance of our algorithms remains asymptotically closer to the AklMaxSINR algorithm.

\section{Comparison of Total Throughput}

By the observation of the experiment, the users with low SINR are the key factor for the total throughput. Because the users with low SINR are the throughput bottleneck and SINR upgrade on throughput improvement is obviously important part for these perceptive users compared to the other users who have high SINR. If we can make the SINR values of these users as good as possible, the total throughput achieve the better performance. The total network throughput is shown in Fig. 5 by using channel overlapping degree in Table 2. The AklMaxSINR is expected to have better performance than the others. The reason is very clear: it is because the AklMaxSINR is a centralized algorithm. Proposed distributed algorithms can achieve a throughput performance like centralized AklMaxSINR.

\section{CONCLUSION}

WLANs have become more and more popular due to their low-cost scalability, convenience, mobility, etc. Most of the APs are distributed over a high density area in an uncoordinated way. Hence, developing an efficient policy to assign channel according to user position will become very crucial to the whole network throughput.

In this paper, we formulate the channel assignment problem as a mathematical model and bring up two distributed algorithms that tries to maximize the sum of weighted SINR, resulting in a higher throughput. Through great quantity simulations, we compared our algorithm to the AklMaxSINR, the Hminmax and the AASC methods. By considering the sum of SINR of the users who are more vulnerable to interference, our distributed algorithms can achieve a performance like centralized AklMaxSINR.

\section{REFERENCES}

[1] A. Mishra, S. Banerjee, and W. Arbaugh, "Weighted coloring based channel assignment for WLANs," ACM SIGMOBILE Mobile Computing and Communications Review, vol. 9, pp. 19-31, 2005.

[2] M. Achanta, "Method and apparatus for least congested channel scan for wireless access points," U.S. Patent 2006/0072602 A1, Apr. 2006.

[3] R. Akl and A. Arepally, "Dynamic channel assignment in IEEE 802.11 networks," in Proc. IEEE Portable 2007 Conf. Portable Information Devices, March 2007, pp. 1-5.

[4] M. Haidar, R. Ghimire, M. A. Rizzo, R. Akl, and Y. Chan, "Channel assignment in an IEEE 802.11 WLAN based on signal-to-interference ratio," in Proc. IEEE CCECE 2008 - Canadian Conference on Electrical and Computer Engineering: Communications and Networking, May 2008

[5] Y. Cui, W. Li, and X. Cheng, "Partially overlapping channel assignment based on "node orthogonality for 802.11 wireless networks," in Proc. IEEE INFOCOM, April 2011, pp.361-365.

[6] X. Jia, L. Xie, Y. Chang, and X. Tang, "Channel assignment for WLAN by considering overlapping channels in SINR interference model," in Proc. IEEE ICNC 2012: Computing, Networking and Communications, Jan 2012, pp. 1005-1009.

[7] A. Mishra, E. Rozner, S. Banerjee, and W. Arbaugh, "Exploiting partially overlapping channels in wireless networks: turning a peril into an advantage," in Proc. 5th ACM SIGCOMM Conf. Internet Measurement, 2005.

[8] E. G. Villegas, E. Lpez-Aguilera, R. Vidal, and J. Paradells, "Effect of adjacent-channel interference in ieee 802.11 wlans," in Proc. CrownCom 2007 Conf. Cognitive Radio Oriented Wireless Networks and Communications, Aug. 2007, pp. $118-125$.

[9] Y. Ding, Y. Huang, G. Zeng, and L. Xiao, "Channel assignment with partially overlapping channels in wireless mesh networks," in Proc. WICON'08 4th Annu. Conf. Wireless Internet, 2008

[10] M. Burton, "Channel overlap calculations for 80211 b networks," White Paper, Cirond Technologies Inc., Tech. Rep., Nov. 2002.

[11] J. G. Proakis and M. Salehi, Digital communications, McGraw-Hill, 5th edition, 2008, pp. 13-14.

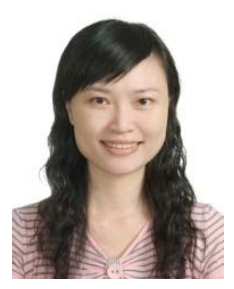

Ya-Yin Yang received the Ph. D. degree in electrical engineering at the National Taiwan University, Taiwan, in 2009. She is currently an Assistant Researcher in the Institute of Computer and Communication Engineering, National Cheng Kung University, Tainan City, Taiwan. Her main research interests include channel estimation, radio resource allocation, and interference cancellation for wireless communication systems.

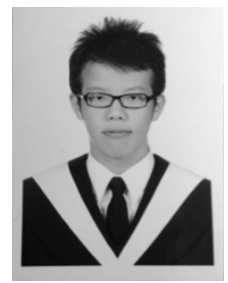

I-Yung Chen received the B.S. degree from the National Pingtung University of Education. He is currently studying the Master degree in electrical engineering at the National Cheng Kung University. His research interests are in wireless communication. 
Chu-Sing Yang is a professor of electrical engineering in the Institute of Computer and Communication Engineering at National Cheng Kung University, Tainan, Taiwan. He received the B.Sc. degree in Engineering Science from National Cheng Kung University in 1976 and the M.Sc. and $\mathrm{Ph} . \mathrm{D}$. degrees in Electrical Engineering from National Cheng Kung University in 1984 and 1987 respectively. He joined the faculty of the Departmen of Electrical Engineering, National Sun Yat-sen University, Kaohsiung, Taiwan, as an Associate Professor in 1988. Since 1993, he has been Professor in the Department of Computer Science and Engineering, National
Sun Yat-sen University. He was the chair of the Department of Computer Science and Engineering, National Sun Yat-sen University from August 1995 to July 1999, and the director of the Computer Center, National Sun Yat-sen University from August 1998 to October 2002. He was the Program Chair of ICS-96 and Program Co-Chair of ICPP-2003 and MTPP-2010. He joined the faculty of the Department of Electrical Engineering, National Cheng Kung University, Tainan, Taiwan, as a Professor in 2006. He participated in the design and deployment of Taiwan Advanced Research and Education Network and served as the deputy director of National Center for High-performance Computing, Taiwan from January 2007 to December 2008. His research interests include future classroom/meeting room, intelligent computing, and network virtualization. 